\title{
How People Write Together Now: Exploring and Supporting Today's Computer-Supported Collaborative Writing
}

\section{Dakuo Wang}

University of California, Irvine

Irvine, CA 92617, USA

dakuow1@uci.edu

\section{Abstract}

Collaborative writing has become increasingly common now. I revisit this research topic because both the tools and people's skills have changed. In my dissertation project, I use both qualitative and quantitative research methods to investigate how people write together now. I have also built information visualization systems to complement existing research methods. By

Permission to make digital or hard copies of part or all of this work for personal or classroom use is granted without fee provided that copies are not made or distributed for profit or commercial advantage and that copies bear this notice and the full citation on the first page. Copyrights for third-party components of this work must be honored. For all other uses, contact the Owner/Author.

Copyright is held by the owner/author(s).

CSCW'16 Companion, February 27 - March 02, 2016, San Francisco, CA USA

ACM 978-1-4503-3950-6/16/02

http://dx.doi.org/10.1145/2818052.2874352 understanding what practices and tools people use in collaborative writing, I propose design implications for system designers to improve the tools, as well as collaboration guidelines for people to write together better.

\section{Author Keywords}

Collaborative Writing; information visualization; computer-supported cooperative work; mixed research methods; Google Docs.

\section{ACM Classification Keywords}

H.5.m. Information interfaces and presentation (e.g., HCI): Miscellaneous.

\section{Introduction}

In recent years, collaborative writing has become more common and important in many professions [6]. The goal of this stream of research is to better understand today's collaborative writing practices with the help of modernized computer systems (e.g., Word and Google Docs), and to generate design implications for improving these tools. Many researchers and designers had explored this question 30 years ago $[1 ; 5 ; 8 ; 10$ 
11], but I believe it is critical to revisit this question now. The two major reasons are: a) People have changed; and b) Technologies have changed tremendously. On the one hand, people's technologyreadiness is much higher than before. People, even young children, are much more comfortable with computers today than they were decades ago. On the other hand, today's technologies are very different than they used to be. Some of the features that support collaboration did not exist back then. Due to these changes, I believe people's practices in collaborative writing are significantly different from what they used to be 30 years ago. Therefore, we need to explore these practices to refresh our knowledge in order to improve the design of existing tools, and to offer collaboration guidelines that adapt to the new technologies.

\section{Related Work}

In the early 1990s, Posner and Baecker were among the first generation of researchers in Human Computer Interaction $(\mathrm{HCI})$ who studied collaborative writing $[1$; $5 ; 8 ; 10 ; 11]$. In 1992, their classic paper, "How people write together", reported the results from interviews of 10 people who had participated in 22 collaborative writing projects [10]. They developed a theoretical framework to describe various aspects of collaborative writing. Lowry et al. [7] extended Posner and Baecker's framework and suggested that the framework should include: collaborative writing activities, document control modes, roles, tools, and work modes. Since then, these understandings and theoretical frameworks have guided the design of many computer systems that support collaborative writing.
Very recently, a few researchers started to revisit the collaborative writing research topic within today's settings. Researchers at Google conducted an internal study to investigate how its employees use Google Docs in collaborative writing [12]. They found that Google Docs is particularly useful for new employees to get acclimated with their group. For example, $70 \%$ of Google's new employees collaborate with others on Google Docs in 2011; and this number raised to $90 \%$ in 2013. Another group of researchers studied how people edit each other's work on Google Docs and they found that co-authors are more likely to comment, rather than to directly change others' words if they are not familiar with each other $[2 ; 3]$. Blau and Caspi studied how people perceive the quality of a co-authored document [4]. From an experiment with 188 college students working in pairs, researchers found that participants thought the others' editing lowered their documents' quality, whereas their contributions increased the documents' quality of the others. Although each of these studies only focused on particular aspects of collaborative writing, they have presented novel findings. This indicates that the developments of technologies enable new ways of writing collaboration, and we should revisit this research topic now.

\section{Problem Statement}

The main research question of this work is how people write together now. In particular, we are interested in the following questions:

- Why do people write collaboratively, and how do they like it?

- What types of documents do people write with others versus writing alone? 


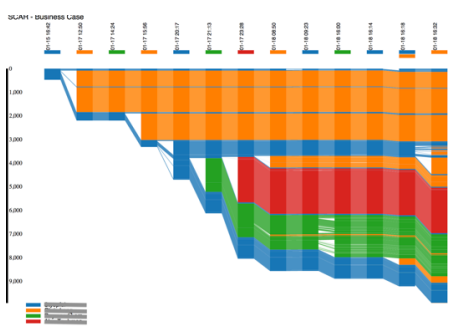

Figure 1: An example of DocuViz visualization

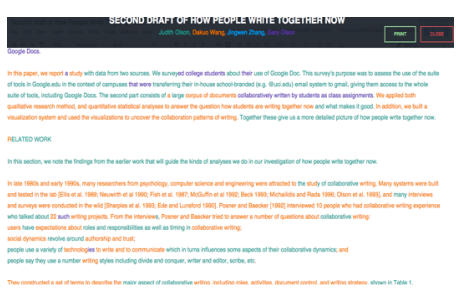

Figure 2: An example of AuthorViz visualization
- What tools and features are people using in their collaborative writing practices, and why do they prefer one tool over the others?

- Would those tools or features help with the work quality or the group dynamics?

- What are the collaboration strategies that people use in collaborative writing, and which strategy can lead to better collaboration (i.e., collaborators can happily produce a higher quality writing in a more efficient way)?

In addition to answering these research questions, I am also interested in addressing them with new research methodologies. Previous ways of studying collaborative writing have been based on traditional qualitative and quantitative methods. But these methods may not be sufficient in this cloud computing era, where millions of people are writing for different purposes and in various scenarios. Thanks to the development of big data techniques and the fact that today's word processors collect traces of user behavior (keystroke by keystroke), I have the opportunity to answer these questions by analyzing at scale. Therefore, we ask the additional question:

- Can we use Big Data techniques (e.g., data mining and information visualization) to facilitate

researchers on collaborative writing, and to support co-authors as well?

\section{Research Methods}

In my dissertation work, I adopt both qualitative and quantitative research methods. I have conducted an indepth interview study of collaborative writing practices of a broad user population (30 participants), including college students, faculty, industrial engineers and researchers, managers, consultants, accountants, and lawyers [14]. In addition to the qualitative research method that relies on what people think they do, I also investigated how people actually write. I collected the data traces, which indicate both authorship and timestamps of users' writing activities, of 96 class assignment documents co-authored by 33 groups of 46 students using Google Docs. Along with my research group, I analyzed the data using quantitative research methods and found that certain features of collaboration (e.g., the evenness of co-authors' contribution) correlated with documents' quality [9]. In addition, by using state-of-the-art natural language processing (NLP), text mining (TM), and information visualization (InfoVis) techniques, I have developed two InfoVis systems (DocuViz [13] and AuthorViz) as a new research approach to facilitate our study. These systems can also help users with their writing collaborations as well.

\section{Dissertation Status}

To date, I have developed two systems, DocuViz and AuthorViz. The first system, DocuViz [13], shows a time-line view of how a Google Doc document evolves over time (Figure 1). The second, AuthorViz, color codes the content showing who contributed what in the final version (Figure 2).

The DocuViz and AuthorViz systems have received positive feedback from both academia and end-users. Together with my research group, I used DocuViz to analyze 96 co-authored documents collected from consenting student teams. From this analysis, we identified new patterns of writing collaborations. We have reported some findings in a peer-reviewed paper [13], and we are developing one more journal paper [9]. As for the end-users, for example, an English 
instructor at Hunter College, NYC, introduced DocuViz to his students of an English writing class. He found the tool to be useful in promoting students' reflective thinking, which helps them improve their writing skills. He shared with us the overall comment that "... many [students] found the maps to give them useful information about how they wrote together - they made the invisible visible ...". Our work has also attracted big companies' attention. Google invited us to give two talks to share with them what we built and found, and encouraged us to develop a more sophisticated version of the tool so that they can use it to analyze the collaborations of the writing of their internal documents.

In addition to the large-scale data-intensive research over what people actually do when they write together now, we also need in-depth qualitative work on what people think they do. Thus I have conducted an indepth interview study on a broad user population about their collaborative writing practices. I found, for example, a number of factors affect how people work and with what technology, such as occupation, scenario, personality, familiarity with the technology, and social status in the group. The study results are currently in submission at CHI 2016 as a full paper.

I am writing my dissertation to wrap up our understandings from the big data research approach, the quantitative study, and the qualitative user study. In my dissertation, I will extend the existing framework on collaborative writing, propose design implications, suggest collaboration guidelines, and embed the good practices into the tools that we developed. This will help collaborators achieve better collaborations in the sense of quality, efficiency, and the relationship between collaborators.

\section{Expected Contribution}

In my continuing analyses, I will contribute to the discussion and exploration surrounding collaborative writing and the practices that new tools enable. This research will form the core of my dissertation, and I plan to disseminate the results through papers on these topics. In addition to the scholarly products, I also plan to apply tailored research results to the design of new tools to support end-users in collaborative writing, and to contribute the tools freely to the public. On an applied level, the results and tools will help in developing new paradigms for coordinating and supporting collaborative writing practices. On a broader level, the results and tools could have implications for designing the next generation technologies related not only to collaborative writing, but also other collaborations, like software development.

\section{Expected Benefits from Attending the \\ Colloquium}

I believe that this colloquium can provide me with a great opportunity to discuss both my dissertation work and future career path with senior faculty and student peers. The colloquium is also a great place to find potential collaborators and future colleagues. I expect to graduate in the summer of 2016, thus I need to make a number of life-changing decisions to make this happen. Therefore, at this stage, I am eager to listen to the senior researchers' advice. I also want to contribute to the HCI research community, such as reviewing other participants' dissertation projects, and providing my knowledge and expertise to them. This colloquium is a great venue to do so. 


\section{Acknowledgements}

I thank all the students and interviewees who participated in our studies. I also thank all members of the Hana Ohana research group, who wrote and provided helpful comments on previous papers and this application. My work is generously supported by a Google Focused Faculty Research Award and a grant from the National Science Foundation (ACI-1322304).

\section{References}

1. Ronald M. Baecker, Nastos Dimitrios, Ilona R. Posner, and Kelly L. Mawby. 1993. The usercentered iterative design of collaborative writing software. In Proceedings of the INTERACT'93 and CHI'93, 399-405.

2. Jeremy Birnholtz and Steven Ibara. 2012. Tracking changes in collaborative writing: edits, visibility and group maintenance. In Proceedings of the ACM 2012 conference on Computer Supported Cooperative Work, 809-818.

3. Jeremy Birnholtz, Stephanie Steinhardt, and Antonella Pavese. 2013. Write here, write now!: an experimental study of group maintenance in collaborative writing. In Proceedings of the $\mathrm{CHI}^{\prime} 13$, 961-970.

4. Ina Blau and Avner Caspi. 2009. What type of collaboration helps? Psychological ownership, perceived learning and outcome quality of collaboration using Google Docs. In Proceedings of the Chais conference on instructional technologies research, 48-55.

5. Paul Dourish, and Victoria Bellotti. 1992. Awareness and coordination in shared workspaces. In Proceedings of the 1992 ACM conference on Computer-supported cooperative work, 107-114.

6. Lisa S. Ede, and Andrea A. Lunsford. 1990. Singular texts/plural authors: Perspectives on collaborative writing. SIU Press.
7. Paul Benjamin Lowry, Aaron Curtis, and Michelle René Lowry. 2004. Building a taxonomy and nomenclature of collaborative writing to improve interdisciplinary research and practice. Journal of Business Communication 41, no. 1 (2004): 66-99.

8. Judith S. Olson, Gary M. Olson, Marianne Storrøsten, and Mark Carter. 1993. Groupwork close up: A comparison of the group design process with and without a simple group editor. ACM Transactions on Information Systems (TOIS) 11, no. 4 (1993): 321-348.

9. Judith S. Olson, Dakuo Wang, Jingwen Zhang, and Gary M. Olson. 2016. How people write together now. In submission.

10. Ilona R. Posner, and Ronald M. Baecker. 1992. How people write together [groupware]. In System Sciences, 1992. Proceedings of the Twenty-Fifth Hawaii International Conference on, vol. 4, 127138.

11. Mike Sharples, J. S. Goodlet, Eevi E. Beck, Charles C. Wood, S. M. Easterbrook, and Lydia Plowman. 1993. Research issues in the study of computer supported collaborative writing. In Computer supported collaborative writing, 9-28. Springer London.

12. Yunting Sun, Diane Lambert, Makoto Uchida, and Nicolas Remy. 2014. Collaboration in the cloud at Google. In Proceedings of the 2014 ACM conference on Web science, 239-240.

13. Dakuo Wang, Judith S. Olson, Jingwen Zhang, Trung Nguyen, and Gary M. Olson. 2015. DocuViz: Visualizing Collaborative Writing. In Proceedings of the 33rd Annual ACM Conference on Human Factors in Computing Systems, 1865-1874.

14. Dakuo Wang, Judith S. Olson, and Gary M. Olson. 2016. Collaborative Writing Tools and Practices. In Submission. 